\title{
Conceptual product design methodology through functional analysis
}

\begin{abstract}
Due to high competitive nature of todays product market, it is essential for conceptual design architecture solution to be derived faster while still maintaining a certain level of innovation to differentiate it from other competing products. This can be a handful task for the development of complex product designs with the current geometrical-based approaches due to plethora of possible physical alternatives to be considered. The search for product design architecture solutions from its functional requirements is more effective as the functional space is comparatively smaller than the physical search space. This allows the design and development effort to be more focused and saves time and resources. With this notion, there is a driving motivation to adapt the functional approach into the conceptual design process to exploit some of its benefits. In this paper, a methodology to derive the product architecture solution from functional approach is discussed and proposed.
\end{abstract}

Keyword: Conceptual design; Functional approach; Product architecture; Product design 